\title{
IN VITRO COMPARATIVE STUDY OF LAVA ULTIMATE CAD/CAM RESTORATIVE SYSTEM IN COMPARISON TO IPS E-MAX PRESS
}

\author{
Ali Y. Dogheim ${ }^{l} B D S$, Ahmed S. El Kady ${ }^{2} P h D$, Mona M. Ghoneim ${ }^{3} P h D$, Maha A. \\ Abdelmotie ${ }^{4} P h D$
}

\begin{abstract}
INTRODUCTION: The increasing demand for esthetics stimulated research in metal-free, tooth-colored restorations. OBJECTIVES: The study compares the biaxial flexural strength, surface roughness and color stability of CAD/CAM nano ceramic optimized composite resin Lava Ultimate material (3M ESPE), pressable ceramic IPS e-max (ivoclar vivadent) and feldspathic porcelain VM7(VITA) MATERIALS AND METHODS: Ninety specimens were used, divided into three groups 30 specimens each: first group of Lava Ultimate discs, second group of E-max Press discs and third group of VM7 discs: each group was divided into three subgroups ten specimens each: 10 specimens were subjected to biaxial flexural strength test using universal testing machine, 10 to surface roughness test using profilometer and 10 for color stability test using spectrophotometer.

RESULTS: On comparing the biaxial flexural strength test there was no significant difference between Lava ultimate group and E-max press, while there was a significant increase in strength in Lava ultimate and E-max press group than the VM7.

As for the surface roughness test there was no significant difference between Lava ultimate and E-max press, while there was a significant increase in roughness in VM7 group than Lava ultimate and E-max press groups.

The Color difference in the three groups showed a significant increase in $\Delta \mathrm{E}$ in Lava ultimate than $\mathrm{E}$-max press. Also, there was a significant decrease in $\triangle \mathrm{E}$ in VM7 group than Lava ultimate and $\mathrm{E}$-max press groups.

CONCLUSIONS: The E-max press and the Lava Ultimate showed significantly higher biaxial flexural strength values and significantly lower surface roughness compared to VM7. Lava Ultimate has the least color stability due to its composite matrix although it could be repolished. VM7 is the most color stable so it could be used as veneers although it is the weakest ceramic in the study. KEYWORDS: Porcelain, biaxial flexural strength, surface roughness, color stability.
\end{abstract}

1- Resident at Conservative Dentistry Department, Faculty of Dentistry, Alexandria University, Alexandria, Egypt

2- Professor of Conservative Dentistry, Faculty of Dentistry, Alexandria University, Alexandria, Egypt.

3- Associate Professor of Conservative Dentistry, Faculty of Dentistry, Alexandria University, Alexandria, Egypt.

4- Associate Professor of Dental Biomaterials Department, Faculty of Dentistry, Alexandria University, Alexandria, Egypt.

\section{INTRODUCTION}

Technical advances in hardware, software and materials provide dentists with new and improved options for indirect restorative materials.

CAD/CAM technologies offer more options to dentists and technicians for fabricating dental restoration. Fueling this trend are the development of intraoral scanning, faster and more accurate milling machines and stronger, tougher CAD/CAM materials (1).

Ceramics inlays/onlays have been developed for their excellent properties in terms of biocompatibility, chemical durability, and optical and esthetic superiority. Ceramics are believed to be superior in reproducing the beauty of form and the harmonious blend of shade and color. However, ceramic inlays have never been adopted for routine widespread use, because of their brittle nature, exacting techniques, time consuming methods, consequential expenses in fabrication and wear of opposing teeth (1).

A new material, called a Resin Nano Ceramic (RNC), is unique in durability and function. The material is a mixture of both resin and ceramic and primarily ceramic. Like a composite resin, the material is not brittle and exhibits high fracture toughness. Like a glass ceramic, the material has excellent polish retention for lasting esthetics. This new material is highly heat cured through a controlled, proprietary manufacturing process, which eliminates the need for a firing step after milling. The material is easily machined chairside or in a dental lab, polishes quickly to an esthetic finish and if necessary, it can also be further adapted using light-cure restoratives (2).

The best example for this material is $3 \mathrm{M}^{\mathrm{TM}}$ ESPETM Lava ${ }^{\mathrm{TM}}$ Ultimate CAD/CAM Restorative it is a "new to the world" CAD/CAM product utilizing 3M's revolutionary nanoceramic technology. Lava Ultimate CAD/CAM blocks perform similarly to or better than glass ceramic and composite materials.

Benefits of Lava Ultimate restorative to the dentist and patient include a faster procedure compared to other CAD/CAM materials, of Durability and shock absorption characteristics from a unique combination of mechanical properties, intra-oral adjustability with light cured restoratives (3).

Another system includes innovative lithium disilicate glass-ceramic materials, which are particularly suited for single restorations as posterior inlay/onlay restorations, and high-stability zirconium oxide materials for long-span bridges it is called IPS e.max which comprises highly esthetic, high-strength materials (4).

The traditional feldspathic porcelain is typically just used for veneers and veneering metal-ceramics. These ceramics are typically crafted using powder and liquid by hand.

Because of different compositions and manufacturing techniques, dental ceramics vary in their physical and mechanical properties. One important property is the strength of the materials, and specially the flexural strength, because of 
the brittle nature of ceramics. Therefore, the aim of this study was to evaluate the biaxial flexural strength of lava ultimate which is composed of resin nano ceramic to that of IPS e-max press and Vita VM7. Also to compare surface roughness as well as color stability of restorative materials (4).

In light of the above information, the null hypothesis to be tested is that the CAD/CAM resin nano ceramic (RNC), will exhibit the same biaxial flexural strength, surface roughness and color stability as pressed ceramics and feldspathic glass ceramics.

\section{MATERIALS AND METHODS \\ Materials:}

1- LavaTM Ultimate CAD/CAM Restorative

\section{Manufacturer 3M ESPE}

Composition: Silica nanomers 20nm, Zirconia nanomers 4$11 \mathrm{~nm}$, Silica-zirconia nanoclusters 0.6-10 micrometers . Fillers $80 \%$ by weight.

Matrix is Bis-GMA, Bis-EMA, UDMA, TEGDMA (5).

2- IPS e.max ${ }^{\circledR}$ Press

Manufacturer Ivoclar Vivadent

Composition: Lithium disilicate crystals (approx. 70\%), Li2Si2O5, embedded in a glassy matrix (6).

3- VM7

Manufacturer Vita Zanhfabrik Bad Sackingen, Germany Composition: Glass frits melted in metal oxides composed of $\mathrm{SIO} 2, \mathrm{AL} 2 \mathrm{O} 3, \mathrm{~B} 2 \mathrm{O} 3, \mathrm{Na} 2 \mathrm{O}, \mathrm{K} 2 \mathrm{O}, \mathrm{CaO}, \mathrm{TiO} 2$ (7).

\section{Methods}

\section{Grouping}

For this study a total of ninety disc shaped specimens were prepared in a split mold with dimentions of $12 \mathrm{~mm}$ in diameter and $2 \mathrm{~mm}$ thickness and divided according to the material used into three groups thirty disc each $(n=30)$ Group I: fabricated from Lava Ultimate. Group II: fabricated from IPS E-max press. GroupIII: fabricated from VM7.

Each group is subdivided into 3 subgroups $(n=10)$ for each test.

\section{Samples construction}

\section{Group I: Lava ultimate samples construction}

DWX dental milling machines (Roland DGA,USA)were used for the milling process of Lava Ultimate blocks into discs according to manufacturer instructions (8).

\section{Group II: IPS E-Max Press samples construction}

A cylindrical split copper mold was designed and constructed to allow the fabrication of wax discs and forming the IPS E-max discs according to manufacturer's instructions (9).

\section{Group III: Fabrication of VM7 ceramic dises:}

VM7 powder and liquid were directly condensed into the hole of the split metallic mould after placement of vita akzent glaze as a separating medium. After drying the specimens were then transferred to the preprogrammed porcelain furnace (Vita programat p300) and the specimens were fired according to the manufacturer's cycle (10).

\section{Biaxial flexural strength test}

Each disc from the previously mentioned groups was centrally loaded on a $10 \mathrm{~mm}$ diameter knife edge support at a cross head speed of $1 \mathrm{~mm} / \mathrm{min}$ with a spherical indenter of $3 \mathrm{~mm}$ diameter using a universal testing machine (Conten Industries Inc. Florida, USA) (11).

A thin section of rubber was placed between the specimen and the knife edge support to ensure uniform loading of the ball indenter on the specimen surface to accommodate any variation in the peripheral thickness or any distortion in the specimens surfaces (12).

The load at failure was recored and the biaxial flexural strength was calculated according to the equation (12):

where $\sigma \max =\frac{P}{h^{[2]}}\left\{(1+v)\left[0.485 \ln \left(\frac{a}{h}\right)+0.52\right]+0.48\right\}$

\section{Where}

$\sigma$ max is the maximum tensile stress (MPa)

$\mathrm{P}$ is the measured load at fracture (Newtons)

$\mathrm{a}$ is the radius of knife edge support ( $\mathrm{mm}$ )

$\mathrm{v}$ is the poisson's ratio for the material

The poisson's ratio of a material is defined as the lateral contraction per unit breadth divided by the longitudinal extention per unit length

(a value of 0.25 was substituted for ceramics and a value of 0.23 was substituted for lava ultimate)

$\mathrm{h}$ is the specimens thickness measured with the digital micrometer ( $\mathrm{mm})$

In is the natural logarithm.

\section{Surface roughness measurements}

All samples were subjected to Laser Scanning Microscopy (LSM).

KEYENCE VK-X100: (Keyence GmbH, Neu-Isenbuerg, Germany) The VK-X Series 3D Laser Scanning Microscope expands the capabilities of laser microscopy combining features of an optical microscope, roughness gauge, laser profilometer, and scanning electron microscope (13).

In this form of optical microscopy, the focused beam of a laser is scanned over the sample and the reflected intensity is displayed as a function of position to create a digital reflected light image of the sample. Scanning a focused laser beam allows the acquisition of digital images with very high resolution since the resolution is determined by the position of the beam rather than the pixel size of the detector. Equipment Confocal laser microscope with red $(633 \mathrm{~nm})$ and IR $(1152 \mathrm{~nm})$ lasers (13).

\section{Color Stability}

The Lava Ultimate specimens were stored in an incubator(US AutoFlow 4000 Series CO2 Water-Jacketed Incubator) in distilled water at $37^{\circ} \mathrm{C}$ for $24 \mathrm{~h}$ for rehydration and complete polymerization following the methodology of previous studies (14).

Baseline colour measurements were made before the immersion in staining solutions (tea). Initial color values were used as a baseline of color change evaluation.

Baseline colors of specimens were measured using VITA Easyshade compact (VITA Zahnfabrik, Bad Sackingen, Germany) (15).

VITA Easyshade was calibrated by placing a probe tip on the calibration port aperture before measurement according to manufacturer's instructions (15). The probe tip was then placed perpendicular at the center of each specimen and flushed into the surface of specimens to obtain accurate measurements. The measurement procedures were repeated three times. All measurements were made on white plexiglass background in order to eliminate background light.

CIE Lab* is expressed by the $\mathrm{L}^{*}$ coordinate representing color luminosity, varying from white to black, and the $\mathrm{a}^{*}$ and $b^{*}$ coordinates representing the chromaticity of the color, with axes varying from green to red and blue to yellow, respectively. Positive a*values cover the red color range and negative values indicate green color range. 
Similarly, positive $b^{*}$ values indicate yellow color range, while negative values indicate blue color range. The means of the values obtained were calculated, and the $\mathrm{L}^{*}, \mathrm{a}^{*}$, and $b^{*}$ parameters were determined. After collection of initial color values, the specimen were immersed in tea for a period of 4 weeks. Specimen were washed thoroughly and dried then subjected to color measurement by CIE L*a*b* (16). The Color difference $\Delta \mathrm{E}$ between the color coordinates was obtained through Hunter equation (17):

$$
\Delta \mathrm{Eab}^{*}=\left[\left(\Delta \mathrm{L}^{*}\right) 2+\left(\Delta \mathrm{a}^{*}\right) 2+\left(\Delta \mathrm{b}^{*}\right) 2\right]^{1 / 2}
$$

The staining solution was renewed every 2 days to avoid bacteria or yeast contamination.

\section{STATISTICAL ANALYSIS}

Data was collected, calculated, tabulated and analyzed using one-way ANOVA Test. Post hoc test was used to compare between groups.

\section{RESULTS}

\section{Biaxial flexural strength}

The biaxial flexural strength values of lava ultimate specimens group I: ranged from 165.86 to $227.99 \mathrm{MPa}$ with mean value of $197.05 \pm 19.62 \mathrm{MPa}$. The biaxial flexural strength test of E-max press specimens group II: ranged from 165.41 to $289.33 \mathrm{MPa}$ as maximum with mean value of $215.3 \pm 37.97 \mathrm{MPa}$. The biaxial flexural strength value of VM7 specimens group III: ranged from 20.29 to $63.23 \mathrm{MPa}$ with mean value of $44.5 \pm 11.83 \mathrm{MPa}$. (Table 1, Figure 1)

Comparison of the mean in the three groups revealed that the differences were statistically significant where the ANOVA test was 132.956 and the p-value was 0.0001 .

Post hoc test between groups reveled that there was no significant difference between Lava ultimate group and E-max press, while there was a highly significant increase in Lava ultimate than VM7, also there was a significant increase in Emax press group than the VM

Table (1): Comparison between the three studied groups regarding Biaxial flexural strength test

\begin{tabular}{|l|c|c|c||}
\hline BFS(MPa) & $\begin{array}{c}\text { Lava } \\
\text { Ultima } \\
\text { te }\end{array}$ & $\begin{array}{c}\text { E-max } \\
\text { press }\end{array}$ & VM7 \\
\hline Min-Max & $\begin{array}{c}165.86- \\
227.99\end{array}$ & $\begin{array}{c}165.41- \\
289.33\end{array}$ & $20.29-63.23$ \\
\hline Median & 198.46 & 206.37 & 46.01 \\
\hline Mean & 197.05 & 215.3 & 44.39 \\
\hline S.D. & 19.62 & 37.97 & 11.83 \\
\hline ANOVA & & \multicolumn{2}{|c|}{132.956} \\
P & & $0.0001^{*}$ \\
\hline P1 & & 0.459 & $0.0001^{*}$ \\
\hline P2 & & & \\
\hline
\end{tabular}

P1 comparison between LU and both E-max and VM7

P2 comparison between E-max and VM7

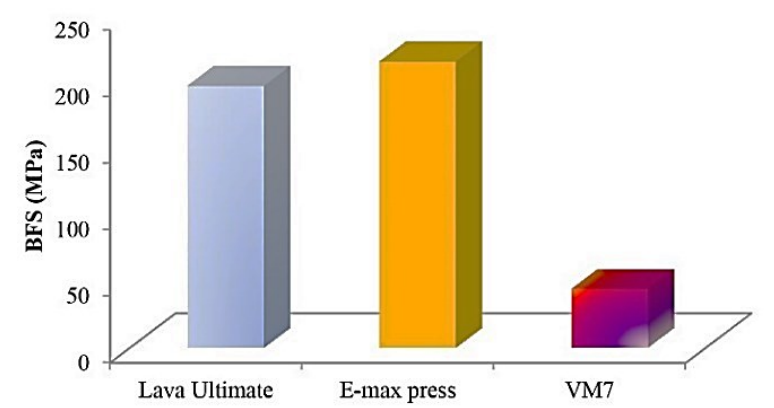

Figure (1): Comparison between the three studied groups regarding Biaxial flexural strength test

\section{Surface roughness test}

The results of the surface roughness test of lava ultimate specimens group I: ranged from 0.43 um to 1.32 um with mean value of $0.899 \pm 0.289 \mathrm{um}$. The results of the surface roughness test of E-max press specimens group II: ranged from 0.48 to $1.33 \mathrm{um}$ with mean value of $0.845 \pm 0.292 \mathrm{um}$. The results of surface roughness test of VM7 specimens group III: ranged from 1.95 to 5.12 um with mean value of $2.826 \pm 0.867 \mathrm{um}$. (Table 2, Figure 2)

Table (2): Comparison between the three studied groups regarding Surface roughness test.

\begin{tabular}{|l|c|c|c||}
\hline SR(um) & $\begin{array}{c}\text { Lava } \\
\text { Ultimate }\end{array}$ & E-max press & VM7 \\
\hline Min-Max & $0.45-1.32$ & $0.48-1.33$ & $1.95-5.12$ \\
\hline Median & 0.9690 & 0.7925 & 2.599 \\
\hline Mean & 0.899 & 0.845 & 2.826 \\
\hline S.D. & 0.2897 & 0.292 & 0.867 \\
\hline $\begin{array}{l}\text { ANOVA } \\
\text { P }\end{array}$ & & 41.439 \\
\hline \multicolumn{1}{|c|}{ P1 } & & $0.0001 * *$ \\
\hline P2 & & 0.925 & $0.0001 *$ \\
\hline
\end{tabular}

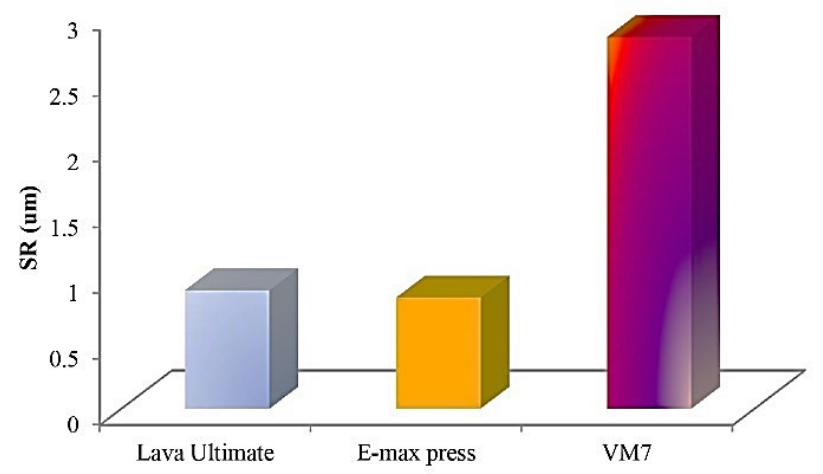

Figure (2): Comparison between the three studied groups regarding Surface roughness test

ANOVA test showed that the difference in means in the three groups were statistically significant where ANOVA test was 41.439 with p-value 0.000 .

Post hoc test between groups reveled that there was no significant difference between Lava ultimate and E-max press, while there was a significant increase in surface 
roughness in VM7 group more than Lava ultimate and Emax press groups. (Figures 3-5)
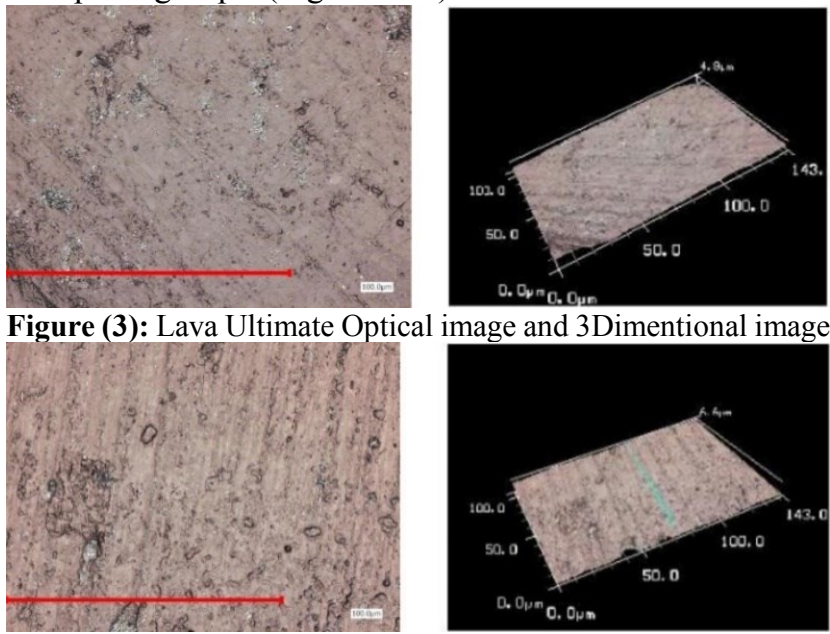

Figure (4): E-max press Optical image and 3Dimentional image

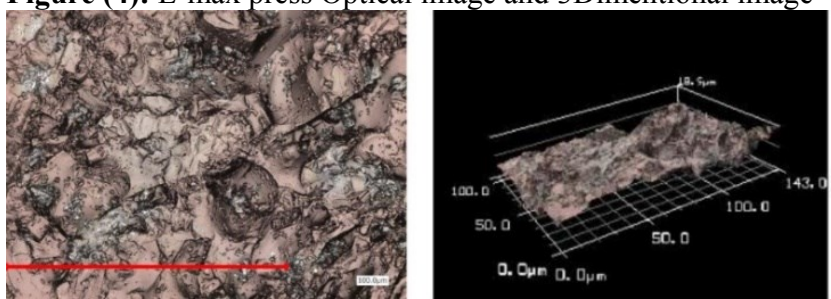

Figure (5): VM7 Optical image and 3Dimentional image

\section{Color stability test results}

The results of the $\Delta \mathrm{E}$ of lava ultimate specimens group I: ranged from 4.398 to 7.44 with mean value of $5.873 \pm 0.923$. The results of the $\Delta \mathrm{E}$ of E-max press specimens group II: ranged from 2.472 to 4.989 with mean value of $3.358 \pm 0.742$. The results of $\Delta \mathrm{E}$ of VM7 specimens group III: ranged from 0.583 to 4.742 with mean value of $1.95 \pm 1.104$. (Table 3, Figure 6)

Table (3): Comparison between the three studied groups regarding change in color

\begin{tabular}{|c|c|c|c|}
\hline$\Delta \mathbf{E}$ & $\begin{array}{c}\text { Lava } \\
\text { Ultimate }\end{array}$ & $\begin{array}{c}\text { E- } \\
\text { max } \\
\text { press }\end{array}$ & VM7 \\
\hline $\begin{array}{l}\text { Min- } \\
\text { Max }\end{array}$ & $\begin{array}{c}4.398- \\
7.440\end{array}$ & $\begin{array}{c}2.472 \\
- \\
4.989\end{array}$ & $0.583-4.742$ \\
\hline Median & 6.055 & 3.168 & 1.770 \\
\hline Mean & 5.873 & 3.358 & 1.955 \\
\hline S.D. & 0.923 & 0.742 & 1.104 \\
\hline $\begin{array}{l}\text { ANOVA } \\
\mathrm{P}\end{array}$ & \multicolumn{3}{|c|}{$\begin{array}{c}45.082 \\
0.0001^{* *}\end{array}$} \\
\hline $\mathrm{P} 1$ & & $0.002 *$ & $0.0001 *$ \\
\hline $\mathrm{P} 2$ & & & $0.001 *$ \\
\hline
\end{tabular}

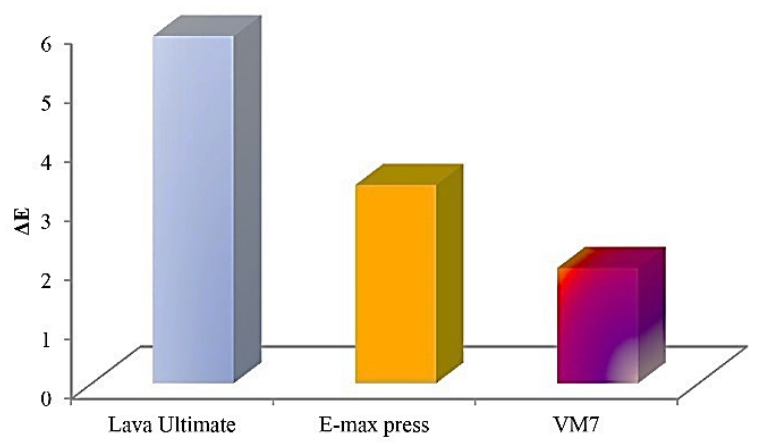

Figure (6): Comparison between the three studied groups regarding change in colour.

Anova test showed that the difference in means in the three groups were statistically significant where Anova test was 45.082 with p-value 0.0001 .

Post hoc test between groups reveled that there was a significant increase in $\Delta \mathrm{E}$ in Lava ultimate than $\mathrm{E}$-max press. Also, there was a significant decrease in $\Delta \mathrm{E}$ in VM7 group more than Lava ultimate and E-max press groups. Which means that VM7 showed the best color stability than E-max press and Lava Ultimate had the least color stability

\section{DISCUSSION}

The in vitro experiments that aim to analyze restoration failures characterized by fracture, surface roughness or color stability are important methods for improving restorative procedures (18).

The main criteria, on which selection of materials was based, was that these three materials can be used as indirect laminate veneers, inlays and onlays although they are different in type and specimen fabrication techniques.

Lava Ultimate $\mathrm{CAD} / \mathrm{CAM}$ restorative materials has high fracture toughness and flexural strength which assure that milled restorations will exhibit excellent durability.

IPS e.max press was selected as a representative of the heat pressable glass ceramics that offers exceptional esthetics, high translucency and a high strength.

Vita VM7 is a fine structured dental ceramic offering the user important advantages, stability characteristics during firing, excellent grinding and polishing properties (18).

The current study compared Lava Ultimate, IPs e.max press and VITA VM7 materials as regards their biaxial flexural strength, surface roughness and colour stability.

Biaxial flexure testing is recognized as a reliable technique and the method of choice (ISO 6872) (12) for studying brittle materials since the maximum tensile stress occurs within the central loading area and edge failures are eliminated. This method was the one recommended by the International Standard Organization because the test standardizes specimen thickness, diameter and shape (19).

In the present study the biaxial flexural strength test of IPS E-max press and Lava ultimate showed significantly higher values than VM7. Similar results were found by Charlton et al. (20), who concluded that flexural strength was significantly higher for a lithia-based ceramic than Vitabloc Mark II which is a feldspathic porcelain. According to Della Bona et al. (21) this result can be explained by greater fracture toughness of the lithia-based ceramic, indicating a potential improvement on the structural performance. Because of its high strength, this 
kind of ceramic is also indicated for anterior fixed partial dentures of up to three elements.

IPS emax press showed higher values than Lava Ultimate but with no significant difference this can be explained by the feature of the ceramics, while the lava ultimate is lower in biaxial flexural strength than IPS emax press as the lava ultimate has a composite matrix while the IPS emax press is pure ceramic and more homogenous material.

The E-max press evaluated in this study presented intermediary flexure values which were significantly higher than the VITA VM7.

With a reported flexural strength of $200 \mathrm{MPa}$, the Lava Ultimate nano-ceramic block has a higher strength than feldspathic porcelain VM7 (22).

This may be due to the structure of the Lava Ultimate which is a recently developed nano-ceramic restorative material formed of unique CAD/CAM blocks based on the integration of nanotechnology and ceramics. The material is said to offer the ease of handling of a composite material with a surface gloss and finish retention similar to a porcelain material (4). Lava Ultimate ${ }^{\mathrm{TM}}$ (3M ESPE) contains a blend of nano-particles agglomerated to clusters and individual bonded nano-particles embedded in a highly cross-linked polymer matrix. It is a combination of aggregated zirconia/silica clusters (composed of $20-\mu \mathrm{m}$ silica and $4-\mu \mathrm{m}$ to $11-\mu \mathrm{m}$ zirconia particles), nonagglomerated/non-aggregated $20-\mu \mathrm{m}$ silica, and nonagglomerated/non-aggregated $4-\mu \mathrm{m}$ to $11-\mu \mathrm{m}$ zirconia with an approximately $80 \%$ ceramic load (4).

Based on the current results, IPS emax pressable ceramics presented the highest flexural strength values of all materials. However, according to the ISO 6872, all of the ceramics used in this study presented flexure strength values that are clinically acceptable the improved strength allows for a somewhat more conservative tooth reduction.

Alberto et al (23) concluded that IPS-e max exhibits significantly higher values in fracture load, flexural strength and hardness than Lava Ultimate ${ }^{\mathrm{TM}}$. On the other hand, the results of our study are in disagreement with the study conducted by Fasbinder (24) who found that, with a reported flexural strength of $200 \mathrm{MPa}$, the nano-ceramic block has a higher initial strength than feldspathic and leucite-reinforced porcelain blocks, as well as veneering porcelains for porcelain-fused-to-metal crowns. (Dennis J. Fasbinder, DDS). However, according to the ISO norm, all of the ceramics presented flexure strength values clinically acceptable, the improved strength allows for a somewhat more conservative tooth reduction.

Roughness is a measure of surface texture and, is often quantified by the deviations of the surface from its ideal form. If the deviations are large, then the surface is considered to be rough; if they are small, then the surface is considered smooth (25).

A confocal laser scanning microscope LSM was used in this study as it has many advantages. It is actually a microscope, scanning electron microscope and roughness Gauge in a Single Unit. It performs profile and roughness measurements with more accuracy and efficiency than these three machines.

As the Laser Scanning Microscope (LSM) is a noncontact laser profilometer so it is more accurate than contact profilometry which underestimate the surface roughness thus present a smoother surface.
In this study the surface roughness of IPS e-max press showed smoother surface than the Lava Ultimate but there was no significant difference. This was in agreement with Wennerberg A et al (26) who found no significant differences between the E-max press and Lava Ultimate groups, they assumed the fact that the reduction in the pores between the grains is not enough to produce a significant difference.

The current study showed significant difference between Lava Ultimate and VM7 group and there was also a significant difference between Emax press and VM7 group. As Emax press is the smoothest followed by Lava Ultimate with no significant difference and the VM7 is the roughest with significant difference between the materials. This was in agreement with Beuer et al. (27) who reported higher strength of $\mathrm{CAD} / \mathrm{CAM}$ veneering ceramic compared to the layered veneering technique. Using of the pressed ceramic may reduce the chipping incidence, since the heat pressing fabrication method would reduce the formation of large surface and flaws present in the bulk of the material.

The strength of a porcelain material was largely determined by its surface roughness and the inner structure of the material may cause a larger stress concentration than that caused by the surface roughness in combination with the surface flaws present on the material.

If the material is given an adequate surface treatment, it will not require properties that stop cracking and the surface of the material would remain smooth, which in turn results in a restoration that will be long lasting.

Several factors including powder compaction, process of forming, firing and also shaping can also cause flaws in ceramics. During these laboratory processes, the flaws may become inherited in the micro structure of the ceramic. Damage caused during grinding; pull-out caused during polishing, micro-porosity present on the subsurface and the introduction of large pores by technicians during restoration manufacture are common causes of technical laboratory flaws that might happen during VM7 fabrication (28).

This would minimize the thermally induced residual (29) stresses. Greater porosities are results of human error that may occur during the fabrication stages in the dental laboratory. Catastrophic failures may also be induced by the incorporation of small impurities like pores, since cracks cannot be healed, but slow growth may occur under oral conditions (30).

The advent of new tooth colored restorative materials within the past three decades has led to a stronger patient orientation toward esthetic dentistry. The patient's demand for esthetic treatment is steadily increasing accordingly, several treatment options have been proposed to restore the esthetic appearance of the dentition, out of which porcelain has been proposed as the most conservative approach. Optimum matching with the surrounding tooth structure depends on not only proper initial color match but also on relative changes that occur with time (31).

In the present study the color stability of IPS e-max press, Lava Ultimate and VM7 was measured when immersed in tea for four weeks.

A spectrophotometer Vita easy shade was used: The CIE Lab was chosen to record color difference $(\Delta \mathrm{E})$ as it is better for the determination of small color differences. Several studies have evaluated various electronic shade matching devices and compared color matching accuracy to human observers (32). 
Seghi et al. (33) used instrumental calorimetric techniques to evaluate the color differences that can exist between different brands of ceramics with identical shade designations. They concluded that the CIE Lab color system provides an objective technique for evaluating the color of dental ceramics.

Spectrophotometric measurements are capable of detecting color differences below the threshold of visual perception. The value of $\Delta \mathrm{E}$ represents relative color changes that an observer might report for the materials after immersion or between time periods. Thus $\Delta \mathrm{E}$ is more meaningful than individual $\mathrm{L}^{*} \mathrm{a} * \mathrm{~b} *$ values (34).

Um and Ruyter (35) explained that both tea and coffee contain yellow colorants. Discoloration by tea due to adsorption of polar colorants onto the surface of the resin composite materials could be removed by brushing the teeth, whereas, discoloration by coffee was due to both absorption and adsorption of the polar colorants onto the surface of materials. This adsorption and penetration of colorants into the organic phase of the materials were explained by the authors as probably being due to the compatibility of the polymer phase with the yellow colorants of coffee.

Immersion time is a critical factor in the color stability. In the present study, color changes become more intensive as immersion time increased. This finding was in accordance with Yannikakis et al (36) who concluded that the most pronounced color change occurred after 30 days and not in the initial hours of immersion time.

These results was in agreement with Ghahramanloo et al. (37) who found that color stability of porcelain (Vita VMK 95) changed after immertion in tea.

The mean $\Delta \mathrm{E}$ of IPS Emax press when immersed in tea for four weeks was 3.3 while that of lava ultimate was 5.8 and the mean $\triangle \mathrm{E}$ of VM7 was 1.9. In other words the VM7 is the most color stable. The IPS e-max press follows it, but Lava Ultimate has the lowest color stability, so color will change noticeably when exposed to stains.

For color matching industrial professionals, an acceptable $\Delta \mathrm{E}$ color difference was the perceptible color difference. Acceptable color differences for dental professionals range from a $\Delta \mathrm{E}$ of 2.6 or 3.3 for in vitro studies, to 3.7 for an in vivo study (38).

Koksal and Dikbas (39) evaluated the color stability of two brands of porcelain teeth. Among the materials tested, porcelain was found to be more resistant to discoloration. They concluded that acrylic teeth showed a higher degree of color change and that the amount of color change for each group increased proportionally with time.

Porcelain was among the initial materials discovered to be used as a definitive anterior esthetic restorative material. In fact its selection was mainly because of its natural appearance, good wear resistance, and color stability. Dental porcelain has color and optical properties that simulate natural teeth. Nevertheless porcelain restorations are considered to be color stable, yet discoloration is one of the major factors for failure of esthetic restorations. There have been several intrinsic or extrinsic factors identified, which are responsible for alterations of surface characteristics and lustre of porcelain. Intrinsic factors involve changes within the material itself and extrinsic factors involve adsorption or absorption of stains in the oral cavity. Literature search has very well showed the range of variation and alteration in color and surface luster of ceramics when artificially exposed with commonly consumed food stuffs and drinks (40).

\section{CONCLUSION}

1- The biaxial flexural strength test of the IPS e max press and the Lava Ultimate showed significantly higher values than VM7.

2- IPS emax press showed higher values than Lava Ultimate but with no significant difference.

3- The surface roughness of both Lava Ultimate and Emax press was significantly lower than VM7 group as VM7 was the roughest.

4- Lava Ultimate has the least color stability due to its composite matrix although it could be repolished every now and then.

5- VM7 is the most color stable so it could be used as veneers although it is the weakest ceramic in the study.

\section{CONFLICT OF INTEREST}

No potential conflict of interest relevant to this article was reported.

\section{REFERENCES}

1. Christensen GJ. In-office CAD/CAM milling of restorations. The future? J Amer Dent Assoc. 2008; 139: 835.

2. Giordano R. Materials for chairside CAD/CAM-produced restorations. J Am Dent Assoc. 2006; 137(Suppl): 14S-21S.

3. Lava ultimate restorative brochure - CEREC-3M. Available http://multimedia.3m.com/mws/media/7561670/lavaultimate-cad-cam-restorativecerec.pdf? fn=lava_ultimate_brochure_cerec-for.

4. Magne P, Kenezevic A. Simulated fracture resistance of composite resin versus porcelain $\mathrm{CAD} / \mathrm{CAM}$ overlay restoration on endodontically treated molar. Quintessence Int. 2009; 40: 125-33.

5. Belli R, Geinzer E, Muschweck A, Petschelt A, Lohbauer U. Mechanical fatigue degradation of ceramics versus resin composites for dental restorations. Dent Mater. 2014; 30 : 424-32.

6. Scientific Documentation IPS e.max ${ }^{\circledR}$ Press. Ivoclar Vivadent. p 6.

7. Queiroz JR, Benetti P, Ozcan M, de Oliveira LF, Della Bona A, Takahashi FE, et al. Surface characterization of feldspathic ceramic using ATR FT-IR and ellipsometry after various silanization protocols. Dent Mater. 2012; 28: 189-96.

8. Alghazzawi TF, Janowski GM. Correlation of flexural strength of coupons versus strength of crowns fabricated with different zirconia materials with and without aging. J Am Dent Assoc. 2015; 146: 904-12.e1.

9. Mohsen C. Corrosion effect on the flexural strength \& micro-hardness of ips e-max ceramics. OJST. 2011; 1: 2935.

10. Puri S. Techniques used to fabricate all-ceramic restorations in the dental practice. Compend Contin Educ Dent. 2005; 26: 519-25; quiz 526-7.

11. Cattel MJ, Knowles JC, Clarke RL, Lynch E. The bi-axial flexural strength of two pressable ceramic systems. J Dent. 1999; 27: 183-96.

12. International Organization for Standardization. ISO 6872: 1995, Dental ceramic. Geneva: ISO; 1995. 
13. Ebeid K, Wille S, Hamdy A, Salah T, El-Etreby A, Kern M. Effect of changes in sintering parameters on monolithic translucent zirconia. Dent Mater. 2014; 30: e419-24.

14. Arocha MA, Mayoral JR, Lefever D, Mercade M, Basilio J, Roig M. Color stability of siloranes versus methacrylateased composites after immersion in staining solutions. Clin Oral Investig. 2013; 17: 1481-7.

15. Chu SJ, Trushkowsky RD, Paravina RD. Dental color matching instruments and systems. Review of clinical and research aspects. J Dent. 2010; 38(Suppl 2): e2-16.

16. Kim-Pusateri S, Brewer JD, Davis EL, Wee AG. Reliability and accuracy of four dental shade-matching devices. J Prosthet Dent. 2009; 101: 193-9.

17. Filho HL, Maia LH, Araújo MV, Eliast CN, Ruellas AC. Colour stability of aesthetic

brackets: ceramic and plastic. Aust Orthod J. 2013; 29: $13-$ 20.

18. Oh SC, Dong JK, Lüthy H, Shärer P. Strength and microstructure of IPS Empress 2 glass-ceramic after different surface treatments. Int J Prosthodont. 2000; 13: 468-72.

19. Sadighpour L, Geramipanah F, Raeesi B. In vitro mechanical tests for modern dental ceramics. J Dent (Tehran). 2006; 3: 143-52.

20. Charlton DG, Roberts HW, Tiba A. Measurement of select physical and mechanical properties of 3 mechinable ceramic materials. Quintessence Int. 2008; 39: 573-9.

21. Buso L, Oliveira-Júnior OB, Hiroshi Fujiy F, Leão Lombardo GH, Ramalho Sarmento H, Campos F, et al. Biaxial flexural strength of CAD/CAM ceramics. Minerva Stomatol. 2011; 60: 311-9.

22. Kassem AS, Atta O, El-Mowafy O. Combined effects of thermocycling and load-cycling on microleakage of computer-aided design/computer-assisted manufacture molar crowns. Int J Prosthodont. 2011; 24: 376-8.

23. Albero A, Pascual A, Camps I, Grau-Benitez M. Comparative characterization of a novel cad-cam polymerinfiltrated-ceramic-network. J Clin Exp Dent. 2015; 7: e495-500.

24. Fasbinder DJ. Nano-Ceramic Restorative Block. A new material is available for chairside CAD/CAM restorations. Inside Dentistry. 2012; 8. Available at: https://www.dentalaegis.com/id/2012/02/nano-ceramicrestorative-block

25. Stachowiak GW, Batchelor AW, Stachowiak GB Experimental methods in tribology. 44th ed. Amsterdam, Oxford: Elsevier, 2004.

26. Wennerberg A, Ohlsson R, Rosen BG, Andersson B. Characterizing three-dimensional topography of engineering and biomaterial surfaces by confocal laser scanning and stylus techniques. Med Eng Phys. 1996;18:548-56.

27. Beuer F, Schweiger J, Eichberger M, Kappert HF, Gernet W, Edelhoff D. High-strength CAD/CAM-fabricated veneering material sintered to zirconia copings - A new fabrication mode for all-ceramic restorations. Dent Mater. 2009; 25: 121-8.

28. Kursoglu P, Karagoz Motro PF, Kazazoglu E. Correlation of surface texture with the stainability of ceramics. J Prosthet Dent. 2014; 112: 306-13.

29. Isgró G, Addison O, Fleming GJ. Transient and residual stresses in a pressable glass-ceramic before and after resin
- cement coating determined using profilometry. J Dent. 2011; 39: 368-75.

30. Stawarczyk B, Ozcan M, Roos M, Trottmann A, Sailer I, Hämmerle CH. Load-bearing capacity and failure types of anterior zirconia crowns veneered with overpressing and layering techniques. Dent Mater. 2011; 27: 1045-53.

31. Bagheri R, Burrow MF, Tyas M. influence of food stimulating solutions and surface finish on susceptibility to staining of aesthetic restorative materials. J Dent. 2005; 33: 389-98.

32. Khokhar ZA, Razzoog ME, Yaman P. Colour stability of restorative resins. Quintessence Int. 1991; 22: 733-7.

33. Seghi RR, Hewlett ER, Kim J. Visual and instrumental colorimetric assessments of small color differences on translucent dental porcelain. J Dent Res. 1989; 68: 1760-4.

34. Yannikakis SA, Zissis AJ, Polyzois GL, Caroni C. Colour stability of provisional resin restorative materials. J Prosthet Dent. 1998; 80: 533-9.

35. Um CM, Ruyter IE. Staining of resin-based veneering materials with coffee and tea. Quintessence Int. 1991; 22: 377-86.

36. Yannikakis SA, Zissis AJ, Polyzois GL, Caroni C. Colour stability of provisional resin restorative materials. J Prosthet Dent. 1998; 80:533-9.

37. Ghahramanloo A, Madani AS, Sohrabi K, Sabzevari S. An evaluation of color stability of reinforced composite resin compared with dental porcelain in commonly consumed beverages. J Can Dent Assoc. 2008;136:673-80.

38. Johnston WM, Kao EC. Assessment of appearance match by visual observation and clinical colorimetry. J Dent Res.1989; 68: 819-22.

39. Koksal T, Dikbas I. Color stability of different denture teeth materials against various staining agents. Dent Mater J. 2008; 27: 139-44.

40. Sulieman M. An overview of tooth discoloration; extrinsic, intrinsic and internalized stains. Dent Update. 2005; 32: 463-4, 466-8,471. 